\title{
CONSUMO E DIGESTIBILIDADE APARENTE DE MINERAIS EM DIETAS PARA CÃES CONTENDO POLPA DE CITROS E FOLHA DE ALFAFA
}

\author{
INTAKE AND APPARENT DIGESTIBILITY OF THE MINERALS IN CANINE \\ DIETS CONTAINING CITRUS PULP AND ALFALFA LEAF
}

\author{
Maria Isabel Fonseca Rocha Malafaia ${ }^{1}$ Marinaldo Divino Ribeiro ${ }^{2}$ \\ Maria Raquel Silva ${ }^{2}$ Pedro Malafaia ${ }^{3}$ Ângela Maria Quintão Lana ${ }^{4}$
}

RESUMO

O consumo diário e a digestibilidade aparente de macro e microminerais, foram determinados em cadelas que receberam uma dieta controle $(R 0)$, acrescida de $10(R 10)$ ou $20 \%$ (R20) de uma mistura contendo polpa de citros $(P C) e$ folhas de alfafa desidratadas. A digestibilidade aparente dos minerais foi estimada por meio da quantidade do elemento mineral ingerido, subtraída daquela eliminada nas fezes. A medida que a ingestão de fibra dietética aumentou, o consumo de $\mathrm{MS}$ (em g/kgPV) diminuiu e as digestibilidades aparentes do $\mathrm{Ca}$, $P, M g, N a, Z n, F e$ e Cu foram maiores. A exceção foi para o Mn, cuja digestibilidade reduziu-se com o aumento da ingestão de fibra. Apenas para o $K$, não se verificou alteração na sua digestibilidade em função do nível de fibra na dieta.

Palavras-chave : fibra dietética, micro e macrominerais, nutrição canina.

\section{SUMMARY}

The daily intake and the apparent digestibility of macro and microminerals were determined in bitches fed either a control diet $(R 0)$ or the same diet but containing $10 \%(R 10)$ or $20 \%$ (R20) of a mixture composed of citrus pulp and dehydrated alfalfa leaf. The apparent digestibility was estimated as being the amount of a mineral element ingested minus the amount of the element quantified in the feces. Once the dietary fiber intake increased, the intake of dry matter (in $\mathrm{g} / \mathrm{kgLW}$ ) reduced and the $\mathrm{Ca}, \mathrm{P}, \mathrm{Mg}, \mathrm{Na}, \mathrm{Zn}, \mathrm{Fe}$ and $\mathrm{Cu}$ apparent digestibility increased. Exception was the Mn digestibility reduction as the fiber intake increased. Only the K digestibility was not influenced by the diet fiber level.
Key words: canine nutrition, dietary fiber, micro and macrominerals.

\section{INTRODUÇÃO}

A ciência da nutrição canina vem desenvolvendo-se e inúmeras dietas, com níveis adequados de nutrientes para as várias classes de tamanho do corpo e fases do ciclo vital, são anualmente lançadas no mercado. Uma dieta balanceada deve conter ingredientes cuja digestibilidade seja elevada, o que resulta em maior aporte de nutrientes para atender à demanda dos tecidos do animal. Dessa maneira, a digestibilidade constitui parâmetro importante na avaliação de dietas para cães.

Até meados dos anos 70, os constituintes da parede celular vegetal (CPV), também conhecidos analiticamente por fibra bruta, fibra em detergente neutro ou por fibra dietética total, foram descritos como componentes inertes nas rações de animais carnívoros e onívoros (EHLE et al., 1982). Entretanto, após inúmeras publicações, essa idéia foi repensada, especialmente no que diz respeito à ação benéfica desses CPV na saúde do epitélio intestinal, na redução dos níveis intestinais de amônia e outras

\footnotetext{
${ }^{1}$ Médico Veterinário, MSc., Universidade Federal Rural do Rio de Janeiro (UFRRJ), 23851-970, Seropédica - RJ.

${ }^{2}$ Estudantes de Zootecnia, UFRRJ.

${ }^{3}$ Zootecnista, Doutor, Professor Adjunto UFRRJ-IZ-DNAP, 23851-970, Seropédica - RJ. Bolsista doCNPq. E-mail: malafeia@ufrij.br. Autor para correspondência.

${ }^{4}$ Engenheiro Agrônomo, Doutor Professor Adjunto UFRRJ/DMAT.
} 
substâncias tóxicas, na facilidade para defecação e na taxa de passagem da digesta pelo trato gastrintestinal (McDOUGALL et al., 1996).

A contínua ingestão de constituintes da parede celular vegetal pode causar alterações na absorção intestinal de minerais. Este fato está relacionado ao teor de fitatos, à quantidade de fibra ingerida e à capacidade de troca catiônica dos grupos amino, carboxil, fenólicos e hidroxilas alifáticas livres presentes na matriz orgânica denominada de fibra dietética (TOMA \& CURTIS, 1986; Van SOEST, 1994).

Embora os efeitos da ingestão de fibra sobre a digestibilidade dos minerais tenham sido estudados em várias espécies, este assunto foi pouco investigado na espécie canina. Em um único estudo, a inclusão de fibra solúvel (pectina) ocasionou uma pequena redução na absorção de ferro em cães (FERNANDEZ \& PHILLIPS, 1982).

Do exposto, com este experimento, objetivou-se estimar o consumo e determinar a digestibilidade aparente de macro e micro minerais de dietas para cães contendo diferentes níveis de fibra dietética.

\section{MATERIAL E MÉTODOS}

O experimento foi conduzido de acordo com um delineamento inteiramente casualizado, utilizando-se dez cadelas SRD, não castradas, vermifugadas, com peso vivo médio inicial de 10,1 $\pm 1,18 \mathrm{~kg}$, com idade média entre dois e três anos, alojadas individualmente em baias cimentadas, conforme recomendações da ASSOCIATION OF AMERICAN FEED CONTROL OFFICIALS (AAFCO, 1999). Cada período experimental constou de 35 dias, sendo 28 para a adaptação às condições experimentais e sete para a coleta de dados. Durante o período de adaptação, os animais foram pesados semanalmente e levados para receber o sol da manhã e exercitarem-se.

Foram feitas três dietas experimentais, utilizando-se uma ração controle (R0), na qual adicionou-se, $10 \%$ (R10) ou 20\% (R20) de fontes de fibra (Tabela 1). Estas fontes, misturadas previamente em iguais quantidades, foram a polpa de citros (PC) e a folha de alfafa desidratada (FA). Este último material, obtido a partir do feno de alfafa comercial, foi manualmente separado entre caules (desprezados) e folhas (FA). A mistura de 50 $\%$ de PC e $50 \%$ de FA objetivou a obtenção de uma fonte de fibra de moderada fermentação intestinal, que é recomendada em estudos anteriores (FAHEY et al., 1992; SUNVOLD et al., 1995).
A ração controle (R0), comprada quinzenalmente em sacos fechados, foi escolhida por ser comercializada rotineiramente nas lojas como ração para cães em regime de mantença. A necessidade individual de ração $(\mathrm{g} / \mathrm{d})$ foi calculada em função da necessidade diária de energia metabolizável $\left(\mathrm{NEM}\right.$, em Kcal/d) $=\mathrm{PV}^{0,67}$ x 145. A necessidade diária de ração (NDR) foi calculada como sendo NDR $(\mathrm{g} / \mathrm{d})=((\mathrm{NEM} \times 100) / 345) \times$ 1,05 ; dos quais 345 equivale ao conteúdo energético médio de 100 gramas das três dietas (Tabela 1) e 1,05 equivale a $5 \%$ de acréscimo.

Antes de ser ministrada aos animais, a ração controle (R0) foi pesada e colocada nos comedouros individuais. Imediatamente, em cada comedouro, foi colocado 0,5 - 0,6 m $\ell$ de água quente $\left(60-70^{\circ} \mathrm{C}\right)$ por grama de ração. Após a ração controle ficar pastosa, nos comedouros dos grupos R10 e R20, foi então, colocado 10 ou $20 \%$ da fonte de fibra, que era misturada com a ração controle, até formar uma massa inseparável. Após esse procedimento, todos os animais receberam sua alimentação. As dietas foram fornecidas duas vezes ao dia, às $8(40 \%)$ e 16 horas $(60 \%)$ e, a água estava sempre fresca e disponível durante todo o período experimental.

As amostragens das dietas e das sobras da alimentação de cada animal foram realizadas diariamente na fase de coleta. Esses materiais foram conservados a $-5^{\circ} \mathrm{C}$. Ao final de cada semana, as amostras individuais foram homogeneizadas e deram origem a uma amostra composta para cada um desses materiais. No período de coleta, as fezes produzidas individualmente, eram imediatamente pesadas e armazenadas a $-5^{\circ} \mathrm{C}$ até serem pré-secas, à $55^{\circ} \mathrm{C}$ por 72 horas em estufa de ventilação forçada. Após a pré-secagem, todos os materiais foram moídos para atingirem granulometria de $1 \mathrm{~mm}$.

Foram feitas determinações (nas dietas, na polpa de citros, na folha de alfafa e nas fezes) dos teores de Cálcio, Fósforo, Magnésio, Potássio, Sódio, Ferro, Zinco, Cobre e Mânganes; segundo os protocolos descritos no manual da ASSOCIATION OF OFFICIAL ANALYTICAL CHEMISTS (AOAC, 1990). As digestibilidades aparentes (Dap.) foram calculadas como sendo Dap. $=((\mathrm{MI}-$ $\mathrm{MF} / \mathrm{MI}) \mathrm{x} 100$; onde MI equivale a quantidade do elemento mineral ingerido; MF é a quantidade do mineral excretada nas fezes (KRONFELD, 1989). A determinação da quantidade dos minerais nas fezes foi determinada pela coleta total das fezes produzidas no período de coleta. Neste estudo, não se coletou a urina dos animais e, dessa forma, não se quantificou a excreção diária de minerais pela urina. A composição das dietas experimentais pode ser observada na tabela 1 . 
Tabela 1 - Composição das fontes de fibra e das dietas experimentais R0, R10 e R20 utilizadas para a determinação do consumo e da digestibilidade dos minerais.

\begin{tabular}{|c|c|c|c|c|c|}
\hline & Polpa de citrus & Folha de alfafa & Ração controle (R0) & $\mathrm{R} 10$ & $\mathrm{R} 20$ \\
\hline Matéria seca $(\mathrm{MS})^{1}$ & 798,5 & 837,8 & 931,7 & 920,3 & 909,0 \\
\hline Matéria orgânica $(\mathrm{MO})^{2}$ & 723,8 & 857,8 & 910,0 & 900,0 & 894,0 \\
\hline Protéina bruta $(\mathrm{PB})^{2}$ & 69,3 & 294,8 & 240,6 & 237,4 & 231,3 \\
\hline Fibra em detergente neutro $(\mathrm{FDN})^{2}$ & 289,2 & 376,2 & 206,1 & 218,8 & 231,4 \\
\hline Extrato etéreo $(\mathrm{EE})^{2}$ & 34,1 & 38,8 & 62,7 & 60,8 & 55,1 \\
\hline Carboidratos totais $(\mathrm{CHT})^{2}$ & -- & -- & 606,7 & 601,8 & 607,6 \\
\hline Energia bruta $(\mathrm{EB})^{3}$ & 3427,0 & 3523,0 & 4466,6 & 4410,3 & 4346,3 \\
\hline Cálcio $(\mathrm{Ca})^{4}$ & 0,72 & 1,15 & 0,83 & 0,84 & 0,85 \\
\hline Fósforo $(\mathrm{P})^{4}$ & 0,06 & 0,14 & 0,47 & 0,44 & 0,40 \\
\hline Magnésio $(\mathrm{Mg})^{4}$ & 0,06 & 1,82 & 1,26 & 1,23 & 1,20 \\
\hline Sódio $(\mathrm{Na})^{4}$ & 0,05 & 0,03 & 1,93 & 1,74 & 1,15 \\
\hline Potássio $(\mathrm{K})^{4}$ & 0,56 & 1,15 & 0,34 & 0,40 & 0,45 \\
\hline Zinco $(\mathrm{Zn})^{5}$ & 87,80 & 67,00 & 372,31 & 342,72 & 313,24 \\
\hline Ferro $(\mathrm{Fe})^{5}$ & 319,01 & 2146,02 & 261,60 & 358,69 & 455,78 \\
\hline Cobre $(\mathrm{Cu})^{5}$ & 4,62 & 7,41 & 8,69 & 8,42 & 8,15 \\
\hline Manganês $(\mathrm{Mn})^{5}$ & 7,91 & 66,96 & 49,10 & 47,93 & 46,76 \\
\hline Energia metabolizável (Emet) ${ }^{3,6}$ & -- & -- & 3498,5 & 3454,0 & 3404,5 \\
\hline
\end{tabular}

$\mathrm{g} / \mathrm{kg}$ de matéria natural

${ }^{2} \mathrm{~g} / \mathrm{kg}$ de MS

${ }^{3} \mathrm{kcal} / \mathrm{kg}$ de MS

${ }^{4} \mathrm{Em} \%$ da MS

${ }^{5} \mathrm{mg} / \mathrm{kg}$ de MS

${ }^{6}$ Calculada de acordo com a fórmula modificada de Atwater $(\mathrm{AAFCO}, 1999) ; \mathrm{EM}(\mathrm{kcal} / \mathrm{kgMS})=(3,5 \mathrm{x} \mathrm{PB}(\mathrm{g}))+(8,5 \mathrm{x} \mathrm{EE}(\mathrm{g})+(3,5 \mathrm{x}$

$\mathrm{CHT}(\mathrm{g})$; onde $\mathrm{CHT}(\mathrm{g})=100-(\mathrm{PB}+\mathrm{MM}+\mathrm{EE})$.

Os parâmetros estudados foram analisados por intermédio da análise de variância e pela utilização do teste "T" para dados pareados, assumindo-se o nível de 5\% de probabilidade para a comparação das médias.

\section{RESULTADOS E DISCUSSÃO}

A ingestão de Cálcio, expressa em g/kg de peso, foi menor no grupo de animais que ingeriram a dieta controle (Tabela 2). Tal fato pode ser explicado pela maior quantidade de Cálcio presente nas folhas de alfafa $(1,15 \%)$, que foram adicionadas nas dietas contendo 10 e $20 \%$ de fibra dietética. A ingestão de Cálcio, neste estudo, foi inferior às verificadas por FAHEY et al. (1992), 0,37g/kgPV e por SUNVOLD et al. (1995), 0,38g/kgPV, para cães também ingerindo dietas contendo fontes de fibra dietética. Essa diferença pode ser explicada pelo fato de as dietas utilizadas por aqueles autores serem do tipo "super-premium", ou seja, de elevada digestibilidade e concentração de nutrientes, inclusive de minerais. A dieta controle usada neste estudo, apresenta uma composição mineral muito próxima dos menores valores recomendados pela AAFCO em 1999.

O consumo de Fósforo foi maior para a dieta controle (Tabela 2). Como o teor de Fósforo nas fontes de fibra é reduzido, houve um efeito de diluição do teor desse elemento quando se adicionou estas fontes na ração controle. Entretanto, a diferença para os valores descritos por FAHEY et al., (1992) 0,19g/kgPV e por SUNVOLD et al., (1995) $0,20 \mathrm{~g} / \mathrm{kgPV}$, não foi superior à $0,072 \mathrm{~g} / \mathrm{kgPV}$ (Tabela 2).

Neste estudo, a relação Cálcio:Fósforo ingerida, expressa em $\mathrm{g} / \mathrm{kg}$ de peso vivo, foi significativamente maior $(\mathrm{p}<0,05)$ à medida que se elevaram os níveis dietéticos de fibra. Os valores encontrados foram 1,76:1; 1,91:1 e 2,12:1, respectivamente, para as dietas controle, R10 e R20. Essas relações são superiores às descritas como adequadas para espécie canina, que poderiam variar de 1,2:1 até 1,4:1, segundo as recomendações encontradas nos manuais do NRC (1985) e da AAFCO (1999).

Não foram detectadas diferenças na ingestão de Magnésio, quando expressa em $\mathrm{g} / \mathrm{kg}$ de peso vivo (Tabela 2). Os valores encontrados neste estudo, foram aproximadamente três vezes superiores aos recomendados $(0,012-0,014 \mathrm{~g} / \mathrm{kgPV})$ no manual da AAFCO de 1999.

A ingestão de Sódio foi maior para a dieta controle do que para a dieta contendo $20 \%$ de fibra dietética (Tabela 2). Isto pode ser explicado, uma vez que as fontes de fibra continham reduzida concentração de Sódio e, ao serem inseridas na dieta controle, propiciaram uma redução do teor de Sódio na dieta R10 e R20 (Tabela 2). O consumo de Sódio 
Tabela 2 - Consumo diário de Matéria Seca (MS), Fibra em Detergente Neutro (FDN), Cálcio (Ca), Fósforo (P), Magnésio (Mg), Sódio (Na), Potássio (K), Zinco (Zn), Ferro (Fe), Cobre (Cu) e Manganês (Mn) nas dietas experimentais R0, R10 e R20.

\begin{tabular}{|c|c|c|c|c|c|c|c|c|c|}
\hline \multirow[b]{3}{*}{$\mathrm{MS}^{1}$} & \multicolumn{6}{|c|}{ Consumo diário } & \multicolumn{3}{|c|}{ Consumo diário (/kg de PV) } \\
\hline & \multicolumn{2}{|c|}{ R0 } & \multicolumn{2}{|c|}{ R10 } & \multicolumn{2}{|c|}{ R20 } & R0 & $\mathrm{R} 10$ & R20 \\
\hline & $322,6^{\mathrm{b}}$ & $(60,4)$ & $318,8^{c}$ & $(61,6)$ & $343,4^{a}$ & $(50,4)$ & $31,9^{\mathrm{a}}$ & $30,9^{b}$ & $30,0^{\mathrm{c}}$ \\
\hline $\mathrm{FDN}^{1}$ & $66,4^{\mathrm{c}}$ & $(12,1)$ & $69,7^{\mathrm{b}}$ & $(13,2)$ & $79,5^{\mathrm{a}}$ & $(11,7)$ & $6,5^{c}$ & $6,7^{\mathrm{b}}$ & $6,9^{\mathrm{a}}$ \\
\hline $\mathrm{Ca}^{1}$ & $2,59^{\mathrm{c}}$ & $(0,54)$ & $2,75^{\mathrm{b}}$ & $(0,51)$ & $2,95^{\mathrm{a}}$ & $(0,43)$ & $0,256^{\mathrm{c}}$ & $0,272^{\mathrm{a}}$ & $0,263^{b}$ \\
\hline $\mathrm{P}^{1}$ & $1,47^{\mathrm{a}}$ & $(0,34)$ & $1,44^{\mathrm{b}}$ & $(0,25)$ & $1,43^{\mathrm{b}}$ & $(0,21)$ & $0,146^{\mathrm{a}}$ & $0,143^{\mathrm{a}}$ & $0,128^{\mathrm{b}}$ \\
\hline $\mathrm{Mg}^{1}$ & $0,39^{\mathrm{b}}$ & $(0,11)$ & $0,41^{\mathrm{b}}$ & $(0,10)$ & $0,44^{\mathrm{a}}$ & $(0,06)$ & $0,039^{\mathrm{a}}$ & $0,041^{\mathrm{a}}$ & $0,039^{\circ}$ \\
\hline $\mathrm{Na}^{1}$ & $0,61^{\mathrm{a}}$ & $(0,14)$ & $0,59^{\mathrm{a}}$ & $(0,11)$ & $0,57^{\mathrm{b}}$ & $(0,08)$ & $0,060^{a}$ & $0,058^{\mathrm{a}}$ & $0,051^{\mathrm{b}}$ \\
\hline $\mathrm{K}^{1}$ & $1.08^{\mathrm{b}}$ & $(0.28)$ & $1,13^{\mathrm{b}}$ & $(0.21)$ & $1.19^{\mathrm{a}}$ & $(0,17)$ & $0,107^{\mathrm{b}}$ & $0.112^{\mathrm{a}}$ & $0.106^{\mathrm{b}}$ \\
\hline $\mathrm{Zn}^{2}$ & $116,1^{\mathrm{a}}$ & $(31,1)$ & $113,6^{\mathrm{b}}$ & $(2,18)$ & $112,1^{\mathrm{b}}$ & $(16,3)$ & $11,49^{\mathrm{a}}$ & $11,25^{\mathrm{a}}$ & $10,01^{\mathrm{b}}$ \\
\hline $\mathrm{Fe}^{2}$ & $81.6^{\mathrm{c}}$ & $(22,4)$ & $1149^{\mathrm{b}}$ & $(21.5)$ & $149.0^{\mathrm{a}}$ & (24.9) & $8.08^{c}$ & $11.37^{\mathrm{b}}$ & $13,30^{\circ}$ \\
\hline $\mathrm{Cu}^{2}$ & $2,71^{\mathrm{c}}$ & $(0,73)$ & $2,78^{\mathrm{b}}$ & $(0,52)$ & $2,86^{\mathrm{a}}$ & $(0,41)$ & $0,268^{a}$ & $0,275^{\mathrm{a}}$ & $0,255^{\mathrm{b}}$ \\
\hline $\mathrm{Mn}^{2}$ & $15,21^{\mathrm{a}}$ & $(4,21)$ & $15,88^{\mathrm{a}}$ & $(2,91)$ & $16,45^{\mathrm{b}}$ & $(2,43)$ & $1,497^{\mathrm{b}}$ & $1,572^{\mathrm{a}}$ & $1,469^{\circ}$ \\
\hline
\end{tabular}

Médias, na mesma linha e seguidas de mesma letra, não diferem em nível de $5 \%$

Números entre parênteses equivalem ao desvio padrão

1 em gramas

${ }^{2}$ em miligramas

foi aproximadamente 3,0 vezes superior e, o de Potássio, 1,6 vezes inferior aos recomendados pela AAFCO em $1999(0,02 \mathrm{~g} / \mathrm{kgPV}$ para o $\mathrm{Na}$ e 0,18 $0,20 \mathrm{~g} / \mathrm{kgPV}$ para o K).

Em função da maior concentração de Zinco na dieta controle (Tabela 2), a inclusão das fontes de fibra dietéticas, as quais continham baixo teor de Zinco, fez com que o consumo deste elemento fosse menor, principalmente na dieta R20 (Tabela 2). Entretanto, mesmo na dieta R20, a ingestão de $\mathrm{Zn} \mathrm{em} \mathrm{mg} / \mathrm{kg}$ de peso vivo, foi superior à recomendada por KRONFELD (1989), cuja recomendação ótima ficaria entre 3,3 e $6,7 \mathrm{mg} / \mathrm{kg}$ de peso vivo.

A ingestão de Ferro foi significativamente influenciada pela adição das fontes de fibra (Tabela 2). Tal fato deve-se à grande quantidade de ferro verificada nas folhas de alfafa desidratada (Tabela 2). A exigência ideal de Ferro se situa entre 2,3 e 3,5 $\mathrm{mg} / \mathrm{kg}$ de peso vivo (KRONFELD, 1989). Dessa forma, os valores encontrados neste estudo, para todos os tratamentos, foram superiores aos considerados ideais.

Para KRONFELD (1989), a ingestão considerada ideal para o Cobre, em $\mathrm{mg} / \mathrm{kg}$ de peso vivo, varia de 0,50 até 1,4 . Os valores observados neste estudo, em teoria, estão próximos da metade do limite inferior da faixa "ótima" (Tabela 2). Entretanto, o manual do NRC (1985), cita valores ideais entre $0,16-0,25 \mathrm{mg} / \mathrm{kg}$ de peso vivo; que são ligeiramente inferiores aos valores verificados neste estudo (Tabela 2). Dessa maneira, as exigências de micro-minerais, consideradas como ideais para a espécie canina, estão longe de um consenso quando se consulta a literatura disponível sobre o assunto.
A maior ingestão de Manganês, verificada na dieta contendo $10 \%$ de fontes de fibra, carece de explicação, uma vez que esta dieta apresentou um teor de Mn intermediário entre as outras dietas (Tabela 2). Porém, os valores observados neste estudo, se situam dentro da faixa "ótima" (1,0 $2,7 \mathrm{mg} / \mathrm{kg}$ de peso vivo) descrita por KRONFELD (1985).

O consumo de MS, em $\mathrm{g} / \mathrm{kgPV}$, diminuiu significativamente $(\mathrm{p}<0,05)$ com o aumento do consumo das fontes de fibra (Tabela 2). Este fato pode estar associado ao acréscimo na digestibilidade aparente do Cálcio, Fósforo e Magnésio nas dietas R10 e R20 (Tabela 3). Tal fato pode ser, em parte, explicado pelo maior tempo de retenção da digesta no trato-gastrintestinal desses animais que comeram as dietas contendo essas fontes de fibra dietética. Os valores de digestibilidade aparente, especialmente para o Cálcio, podem variar de $0-90 \%$ (CASE $\boldsymbol{e t}$ al., 1995). Tais discrepâncias são atribuídas à origem dos componentes das dietas (fontes vegetais possuem mais fitatos do que as de origem animal), ao consumo de MS, a idade dos animais (animais mais velhos reduzem sua capacidade de absorver $\mathrm{Ca}$, $\mathrm{P}$ e $\mathrm{Mg}$ ) e à concentração de vitamina $\mathrm{D}$ na dieta. Dessa forma, os valores encontrados neste estudo podem ser considerados normais.

Para o Sódio e o Potássio, os valores foram numericamente superiores aos verificados para os outros minerais (Tabela 3). Isto é similarmente documentado na literatura e, deve-se ao fato de serem encontrados em formas solúveis e de fácil dissociação molecular no tubo digestivo. 
Tabela 3 - Digestibilidade aparente do Cálcio (Ca), Fósforo (P), Magnésio (Mg), Sódio (Na), Potássio (K), Zinco (Zn), Ferro $(\mathrm{Fe})$, Cobre $(\mathrm{Cu})$ e Manganês $(\mathrm{Mn})$ nas dietas experimentais R0, R10 e R20.

\begin{tabular}{|c|c|c|c|c|c|c|}
\hline \multirow[b]{3}{*}{$\mathrm{Ca}$} & \multicolumn{6}{|c|}{ Digestibilidade aparente $(\%)$} \\
\hline & \multicolumn{2}{|c|}{ R0 } & \multicolumn{2}{|c|}{ R10 } & \multicolumn{2}{|c|}{ R20 } \\
\hline & $30,2^{\mathrm{c}}$ & $(4,8)$ & $35,6^{\mathrm{a}}$ & $(2,6)$ & $34,8^{\mathrm{a}}$ & $(1,9)$ \\
\hline $\mathrm{P}$ & $32,5^{\mathrm{c}}$ & $(5,3)$ & $35,6^{\mathrm{b}}$ & $(3,6)$ & $45,3^{\mathrm{a}}$ & $(2,6)$ \\
\hline $\mathrm{Mg}$ & $33,6^{\mathrm{c}}$ & $(5,2)$ & $36,2^{\mathrm{b}}$ & $(4,9)$ & $37,8^{\mathrm{a}}$ & $(3,8)$ \\
\hline $\mathrm{Na}$ & $64,2^{\mathrm{c}}$ & $(2,7)$ & $65,9^{\mathrm{b}}$ & $(1,9)$ & $68,3^{a}$ & $(1,9)$ \\
\hline $\mathrm{K}$ & $86,4^{\mathrm{a}}$ & $(1,6)$ & $84,6^{\mathrm{a}}$ & $(1,4)$ & $87,1^{\mathrm{a}}$ & $(1,5)$ \\
\hline $\mathrm{Zn}$ & $25,9^{\mathrm{c}}$ & $(5,3)$ & $27,2^{\mathrm{b}}$ & $(4,1)$ & $30,3^{\mathrm{a}}$ & $(2,5)$ \\
\hline $\mathrm{Fe}$ & $25,8^{\mathrm{b}}$ & $(5,2)$ & $27,4^{\mathrm{a}}$ & $(4,2)$ & $27,8^{\mathrm{a}}$ & $(1,3)$ \\
\hline $\mathrm{Cu}$ & $26,1^{\mathrm{c}}$ & $(5,5)$ & $27,7^{\mathrm{b}}$ & $(3,0)$ & $33,9^{\mathrm{a}}$ & $(4,2)$ \\
\hline $\mathrm{Mn}$ & $22,8^{\mathrm{a}}$ & $(5,1)$ & $19,5^{b}$ & $(4,7)$ & $14,1^{\mathrm{c}}$ & $(2,8)$ \\
\hline
\end{tabular}

Médias, em mesma linha e seguidas de mesma letra, não diferem em nível de $5 \%$;

Números entre parênteses equivalem ao desvio padrão

Para os microminerais, com exceção do Manganês, a medida que se elevaram os níveis de fibra dietética, as digestibilidades aparentes também aumentaram (Tabela 3). Os valores para a digestibilidade do $\mathrm{Mn}$ encontrados na literatura, variam de 40 - 70\%; portanto, os dados deste estudo são muito inferiores aos normalmente descritos.

KRONFELD (1989) cita as amplitudes de $18-65 \%, 4-32 \%$ e $3-26 \%$ para as digestibilidades aparentes, respectivamente, de $\mathrm{Zn}$, $\mathrm{Fe}$ e $\mathrm{Cu}$. Dessa maneira, os dados encontrados neste experimento estão situados dentro destas amplitudes (Tabela 3).

O acréscimo nos valores das digestibilidades aparentes dos minerais a medida que a ingestão de fibra aumentou, não foi relatado na literatura consultada sobre adição de fontes de fibra na alimentação de cães. Entretanto, foram os nutricionistas humanos que fizeram, nos anos 70 , a especulação que haveria maior perda fecal de minerais à medida que houvesse maior ingestão de fibra dietética. Essa especulação não foi suportada pelas evidências experimentais e os resultados foram bastante variáveis; por exemplo, quando a fonte de fibra era a couve ou o repolho, verificouse melhor absorção de $\mathrm{Mg}, \mathrm{Zn}$ e $\mathrm{Cu}$ (Van SOEST, 1994).

\section{CONCLUSÕES}

Conforme verificado neste estudo, à medida que o consumo de MS diminui (em g/kgPV) e a ingestão de fibra aumenta, as digestibilidades aparentes do $\mathrm{Ca}, \mathrm{P}, \mathrm{Mg}, \mathrm{Na}, \mathrm{Zn}, \mathrm{Fe}$ e Cu também aumentam. Entretanto, o coeficiente de digestibilidade do $\mathrm{Mn}$ reduz-se à medida que os animais ingerem mais fibra. Apenas para o K, não se verifica alteração na sua digestibilidade em função do nível de fibra na dieta.

\section{REFERÊNCIAS BIBLIOGRÁFICAS}

ASSOCIATION AMERICAN FEED CONTROL OFFICIALS (AAFCO). AFCO Official Publication. Atlanta, 1999. $162 \mathrm{p}$.

ASSOCIATION OF OFFICIAL ANALYTICAL CHEMISTS. AOAC. Official methods of analysis. 15.ed. Arlington: 1990. V.I, $1117 \mathrm{p}$.

CASE, L.P., CAREY, D.P., HIRAKAWA, D.A. Canine and feline nutrition. Saint Louis : Mosby Year Book, 1995. $455 \mathrm{p}$.

EHLE, F.R., ROBERTSON, J.B., VAN SOEST, P.J. I nfluence of dietary fibers on fermentation in the human large intestine. Journal of Nutrition, v.112, n.1, p.158$166,1982$.

FAHEY, G.C., MERCHEN, N.R., CORBIN, J.E., et al. Dietary fiber for dogs. III. Effects of beet pulp and oat fiber additions to dog diets on nutrient intake, digestibility, metabolizable energy, and digesta mean retention time. Journal of Animal Science, v.70, n.4, p.1169-1174, 1992.

FERNANDEZ, R., PHILLIPS, S.F. Components of fiber impair iron absorption in the dog. American Journal of Clinical Nutrition. v.35, n.1, p.107-115, 1982.

KRONFELD, D.S. Vitamin and mineral supplementaition for dogs and cats. A monograph on micronutrients. California: Veterinary practice, 1989. 127p.

McDOUGALL, G.J., MORRISON, I.M., STEWART, D., et al. Plant cell walls as dietary fiber: Range, structure, processing and function. Journal of Science Food and Agriculture, v.70, p.133-150, 1996.

NATIONAL RESEARCH CONCIL (NRC). Nutrients requeriments of dogs. Washington, DC.: National Academy of Science, 1985. p.123.

SUNVOLD, G.D., FAHEY, G.C., MERCHEN, N.R., et al. Dietary fiber for dogs. IV. In vitro fermentation of selected fiber sources by dog fecal inoculum and In Vivo digestion and metabolism of fiber-supplemented diets. Journal of Animal Science, v.73, n.6, p.1099-1109, 1995.

TOMA, R.B., CURTIS, D.J. Dietary fiber: Effects on mineral bioavailability. Food Technology v.40, n.1, p.111-116, 1986.

Van SOEST, P.J. Nutritional ecology of the ruminant. Ithaca: Cornell University, 1994. 474p. 